\title{
Optical correlation technique for cement particle size measurements
}

\author{
Mykhaylo P. GORSKY*, Peter P. MAKSIMYAK, ANDRew P. MAKSIMYAK \\ Correlation Optic Department, Chernivtsi National University, 2 Kotsybunska St., Chernivtsi, Ukraine \\ ${ }^{*}$ Corresponding author: misha@itf.cv.ua
}

\begin{abstract}
Optical correlation technique of determining the cement particle size distribution is described. It is based on transverse coherent function measurement using a polarization transverse shearing interferometer. The proposed technique of data processing decreases the dependence of the result on interferometer noise, emission source intensity fluctuations and difference of refractive index magnitudes of different cement particles. The technique allows fast and reliable determination of the size distribution function of cement particles.
\end{abstract}

Keywords: transverse coherence, polarization interferometer, cement, size distribution function.

\section{Introduction}

The size distribution function of particles is an important characteristic of cement. Different kinds and brands of cement differ by particle sizes. For physical and mathematical modelling of different processes, which occur during specific hydration and studying its mechanical, optical and other properties, it is necessary to know the size distribution function of particles. Cement particle size distribution measurements are taken by different methods, such as electrical zone sensing, sedimentation, scanning electron microscopy [1-5]. But these methods are too complicated and are not widely used. A standard procedure consists in measuring the weight of the remains on the sieve during consecutive screening from the biggest mesh aperture to the smallest one. Laser light diffraction method [5] is also used. For determination of cement particle size we suggest using optical correlation technique $[6,7]$.

Cement is a complicated mixture of particles with different sizes and forms, which by $95-97 \%$ consists of oxides $\mathrm{CaO}, \mathrm{SiO}_{2}, \mathrm{Al}_{2} \mathrm{O}_{3}$ and $\mathrm{Fe}_{2} \mathrm{O}_{3}$. These compounds constitute minerals, the main of which are [1-4]:

- tricalcium silicate (alite), $3 \mathrm{CaO} \cdot \mathrm{SiO}_{2}, 40-65 \%$;

- dicalcium silicate (belite), $2 \mathrm{CaO} \cdot \mathrm{SiO}_{2}, 15-45 \%$;

- tricalcium aluminate, $3 \mathrm{CaO} \cdot \mathrm{Al}_{2} \mathrm{O}_{3}, 4-12 \%$; 
- tetracalcium alumoferrite, $4 \mathrm{CaO} \cdot \mathrm{Al}_{2} \mathrm{O}_{3} \cdot \mathrm{Fe}_{2} \mathrm{O}_{3}, 12-25 \%$;

- gypsum.

The size of a particle with complex shape could be determined by the maximum linear dimension or by the spherical particle diameter with equivalent volume. As is known, optical properties are mathematically calculated only for spherical, cylindrical and spheroid forms $[8,9]$. For practical use, in calculations of specific cement optical properties, it is convenient to approximate particles by spherical form [10]. This approximation is used in laser radiation diffraction method [5]. But the distribution found essentially depends on incoming parameters for calculation. One of these parameters is the magnitude of relative refractive index. The refractive index of cements is complex $m=n+i \chi$, but its real and imaginary parts are found within the intervals of $n=1.5-1.7, \chi=0.003-1$ [1-5]. As cement is a mixture of particles with different chemical composition, each particle could have its own refractive index magnitude. In the case of measurement of laser radiation diffraction on isolated particles, consideration of this peculiarity is quite complicated. Usually, some refractive index is set for all particles, which causes distortion of results.

It has been shown in papers $[6,7]$ that it is possible to determine sizes and concentration of particles from the transverse coherent function of a particle's image. However, this technique needs high measurement accuracy of coherence function, and samples must provide only single scattering (distances between particles have to exceed maximum particle size). Often in practice, these conditions are hard to provide. We suggest the technique of processing and approximation of experimental results, which decreases the obtained particle size distribution function dependence on concentration and spread in refractive index magnitudes.

\section{Coherence function calculation}

Under light scattering on large particles, particle images are projected into observation plane. It is easy to analyze particle images using the transverse coherence function:

$$
\Gamma_{\perp}(\rho)=\psi_{\perp}(\rho)+\Gamma_{X}
$$

where $\psi_{\perp}(\rho)$ - transverse coherence function of particle images giving the data on particle size distribution; $\Gamma_{X}$ - constant component describing non-scattered radiation intensity. Experimentally, the coherence function of a field is determined in transverse shearing interferometer. The transverse coherence function magnitudes for certain transversal shares are determined from interference pattern visibility. For scalar optical fields visibility is found as:

$$
V=\frac{I_{\max }-I_{\min }}{I_{\max }+I_{\min }}
$$

where $I_{\max }$ and $I_{\min }-$ maximum and minimum magnitudes of intensity, respectively. 


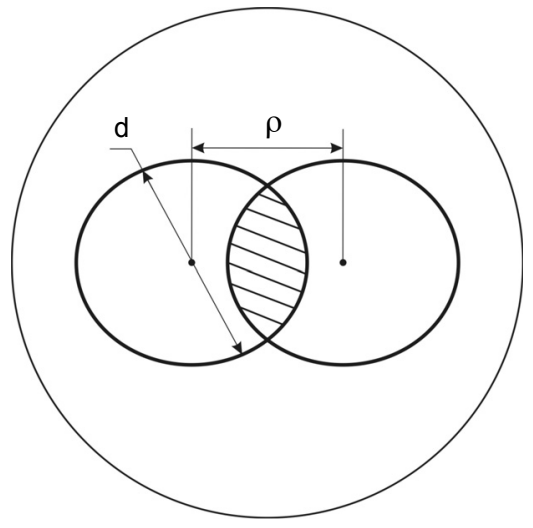

Fig. 1. Image overlay for spherical particle with diameter $d$ and transverse displacement $\rho$.

Let us find the transversal coherence function of spherical particle's image with diameter $d$. The correlation of particle images for transversal displacement in interferometer leg $\rho$ can be defined by overlapped zone square of particle image (Fig. 1):

$$
s_{r}(d, \rho)=\frac{d^{2}}{2} \operatorname{acos} \frac{\rho}{d}-\frac{\rho}{2} \sqrt{d^{2}-\rho^{2}}
$$

Overlapped square normalization of particle images gives us the correlation function of image particle. Let us denote the radiant flux through a unit area by $d I$. The particle image overlaps radiant flux in the beam. Then, in interference maximum, the radiant flux at the observation plane behind the particle image would be the result of beam interference with zero phase difference, and the total radiant flux would be equal to $4 d I$. Radiant flux in particle image area outside the overlapped image zone would be equal to $d I$. The flux in overlapped zone is zero. Particle image zone square, outside the overlapped zone, equals $2\left[d^{2} \pi / 4-s_{r}(d, \rho)\right]$. If the observation zone square is larger than the particle image square by $\rho_{s}$ times, then the observation zone square, without particle images, is determined as: $\rho_{s}\left(d^{2} \pi / 4\right)-2\left[d^{2} \pi / 4-s_{r}(d, \rho)\right]-s_{r}(d, \rho)$. Then, the maximum intensity for displacement $\rho$ and particle diameter $d$ could be written as:

$$
I_{\max }\left(\rho, d, \rho_{s}\right)= \begin{cases}d I\left[d^{2} \operatorname{acos} \frac{\rho}{d}+d^{2} \pi\left(\rho_{s}-\frac{3}{2}\right)-\rho \sqrt{d^{2}-\rho^{2}}\right], & \rho \leq d \\ d I \cdot d^{2} \pi\left(\rho_{s}-\frac{3}{2}\right), & \rho>d\end{cases}
$$

where $\rho_{s}$ - the ratio of the observation field square to the particle image square. In interference minimum, the observation field would be the result of beam interference with phase difference $\pi$, so that the radiant flux outside the particle images and in overlapped image zone equals zero. The radiant flux in not overlapped zones of particle 
images is also equal to $d I$. Then, the minimum intensity in the transverse shearing interferometer for displacement $\rho$ and particle diameter $d$ is:

$$
I_{\min }(\rho, d)= \begin{cases}d I\left[\frac{d^{2} \pi}{2}+\rho \sqrt{d^{2}-\rho^{2}}-d^{2} \operatorname{acos} \frac{\rho}{d}\right], & \rho \leq d \\ d I \frac{d^{2} \pi}{2}, & \rho>d\end{cases}
$$

Then, the transverse coherence function through the extreme magnitudes of intensity is:

$$
\begin{aligned}
\Gamma_{\perp}\left(\rho, d, \rho_{s}\right) & =\frac{I_{\max }-I_{\min }}{I_{\max }+I_{\min }}= \\
& = \begin{cases}\frac{d^{2} \pi\left(\rho_{s}-2\right)-2 \rho \sqrt{d^{2}-\rho^{2}}+2 d^{2} \operatorname{acos} \frac{\rho}{d}}{d^{2} \pi\left(\rho_{s}-1\right)}, & \rho \leq d \\
\frac{\rho_{s}-2}{\rho_{s}-1}, & \rho>d\end{cases}
\end{aligned}
$$

For the ensemble of particles with different sizes and the corresponding size distribution $p(d)$, the transverse coherence function is defined as:

$$
\Gamma_{\perp}^{\text {total }}\left(\rho, \rho_{s}\right)=\int_{0}^{\infty} p(d) \Gamma_{\perp}\left(\rho, d, \rho_{s}\right) \mathrm{d} d
$$

Determination of the distribution function from these equations is very complicated. But, if we know the function $p(d)$, we could find its parameters using the least-squares method [11].

If an analytical form of function $p(d)$ is known, then determination of its parameters is done by the following algorithm. From experimental data, by extreme magnitudes of intensity, the transverse coherence function magnitudes are determined for certain transverse shifts as interference pattern visibility. Then, using the least-squares method and Eq. (7), the best approximation is found, from which distributive parameters and value $\rho_{s}$ could be determined, which gives us particle concentration.

\section{Measuring technique with polarization transverse shearing interferometer}

For measuring the field's transverse coherence function, we use a polarization interferometer arrangement (Fig. 2). It consists of two identical wedges 3 and 4 


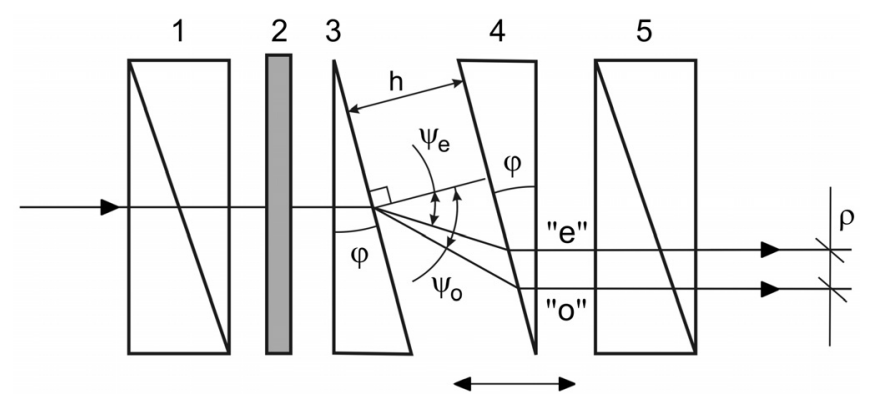

Fig. 2. Beam paths in interferometer.

forming a plane-parallel plate, which are placed between crossed polarizers 1 and 5 . The main optical axes of wedges 3 and 4 are parallel and form $45^{\circ}$ angles with plane of polarization of polarizers 1 and 5. Sample 2 is placed between polarizer 1 and wedge 3 .

In Figure 2, the ordinary "o" and extraordinary "e" beam paths are shown. Space division of the beams occurs on a way out from the first wedge 3 . At normal incoming beam incidence on the surface of wedge 3 , refraction angles of ordinary $\psi_{o}$ and extraordinary $\psi_{e}$ beams could be written as:

$$
\begin{aligned}
& \sin \psi_{o}=\frac{n_{o}}{n} \sin \varphi \\
& \sin \psi_{e}=\frac{n_{e}}{n} \sin \varphi
\end{aligned}
$$

where $\varphi$-incident angle, which is equal to prism angle; $n_{o}$ and $n_{e}$ - refractive indices of ordinary and extraordinary beams, respectively; $n$-refractive index of a surrounding medium.

Transverse displacement between beams $\rho$ is assigned by the distance between wedges $h$ and depends on wedge angle and birefringence of the wedge. From the geometry of Fig. 1 one gets:

$$
\rho=h\left(\tan \psi_{o}-\tan \psi_{e}\right) \cos \varphi=a h
$$

As one can see, $\rho$ is linearly dependent only on $h$ (parameters $\varphi, \psi_{o}, \psi_{e}$ are constant for specific scheme realization). So, for transverse displacement determination it is necessary to know the dependence $\rho=f(h)=a h$.

In the scheme of Fig. 2, transverse displacement $\rho$ is accompanied by longitudinal one, $\rho_{\|}$, determined from the equation:

$$
\rho_{\|}=\left[\left(\frac{1}{\cos \psi_{o}}-\frac{1}{\cos \psi_{e}}\right)-n_{e}\left(\tan \psi_{o}-\tan \psi_{e}\right) \sin \varphi\right] h=b h
$$




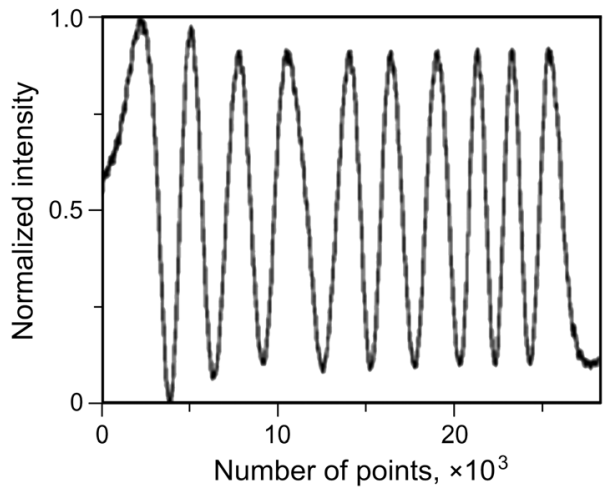

Fig. 3. Intensity changes measured $v s$. longitudinal displacements of interferometer wedges.

Longitudinal displacement between the beams in an interferometer causes modulation of the field intensity, whose dependence, while changing the distance between the wedges from zero to a defined value, is represented in Fig. 3. As longitudinal displacement between beams is linearly bound with transverse one, it is convenient to calibrate transverse displacement with the extreme magnitudes of total field intensity for longitudinal displacements (Fig. 3). The distance between the extrema (maximum and minimum) amounts to $\lambda / 2$. Such a calibration could be provided in case of substantial scale excess of longitudinal field modulation over transverse modulation scale. So, even for irregular changes of the distance between wedges (Fig. 3), the magnitude of transverse displacement is known by extrema of the total field intensity at the output of an interferometer. In our experiment, the distance between neighbouring extrema corresponded to transverse displacement by $3.36 \mu \mathrm{m}$.

\section{Experiment}

For the least-squares method implementation, it is necessary to know function $p(d)$. The analytic function $p(d)$ is not known. That is why in order to find this function we have investigated the images of cement particle samples. The samples consisted of

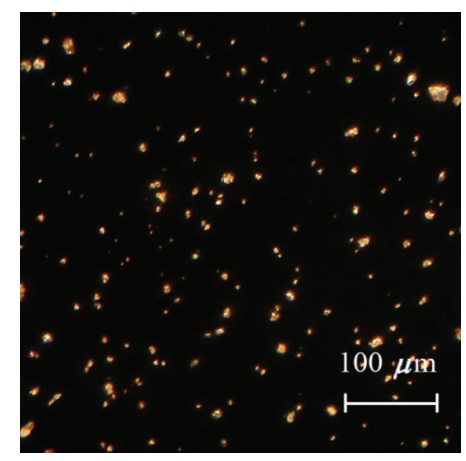

Fig. 4. Microscopic images of cement particles. 


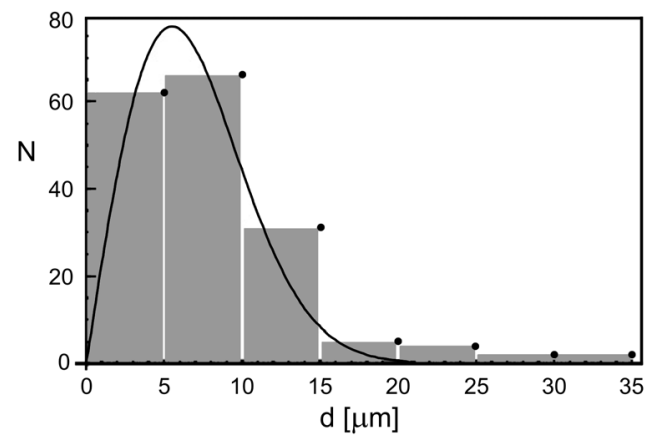

Fig. 5. Calculated dependence of the quantities of cement particles vs. size (bar graph) and Rayleigh distribution approximation (line).

dry cement particles uniformly distributed on a glass plate surface. These samples were later used for measurements in transversal shearing interferometer.

An example of microscopic images of cement particles is shown in Fig. 4. In order to find the analytical view of function $p(d)$ for cement, we have analyzed microscopic sample images. Calculated from images the quantities of cement particles, in corresponding size interval are best approximated by Rayleigh distribution (Fig. 5):

$$
p_{r}(d, \sigma)=\frac{d}{\sigma^{2}} \exp \left(-\frac{d^{2}}{2 \sigma^{2}}\right)
$$

where $\sigma$ - the most probable magnitude of particle diameter.

For measurement purposes we need to obtain images of cement particles. We use an optical scheme shown in Fig. 6. The beam from laser 1, through inverse telescopic system 2, illuminates sample 3 . Images of cement particles 3 , through polarization interferometer 4 , using a microobjective 5 , are projected on a photodetector 6 . Signal from a photodetector is recorded by computer 7 .

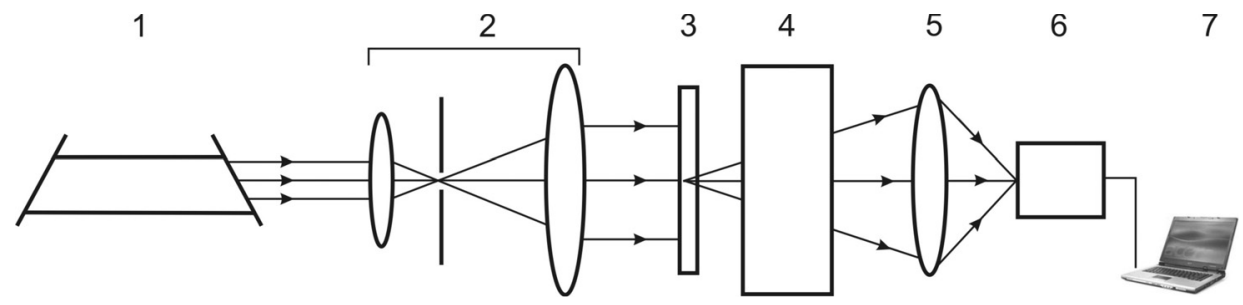

Fig. 6. Experimental optical scheme: 1 - laser, 2 - inverse telescopic system with diaphragm, 3 - sample, 4 - polarization interferometer, 5 - microobjective, 6 - photodetector, 7 - computer.

Measurement precision was controlled each time by a microscope. Measurements were performed for samples with high and low concentration of cement particles. Typical experimental dependences of intensity obtained from measurement using interferometer are shown in Fig. 7. 

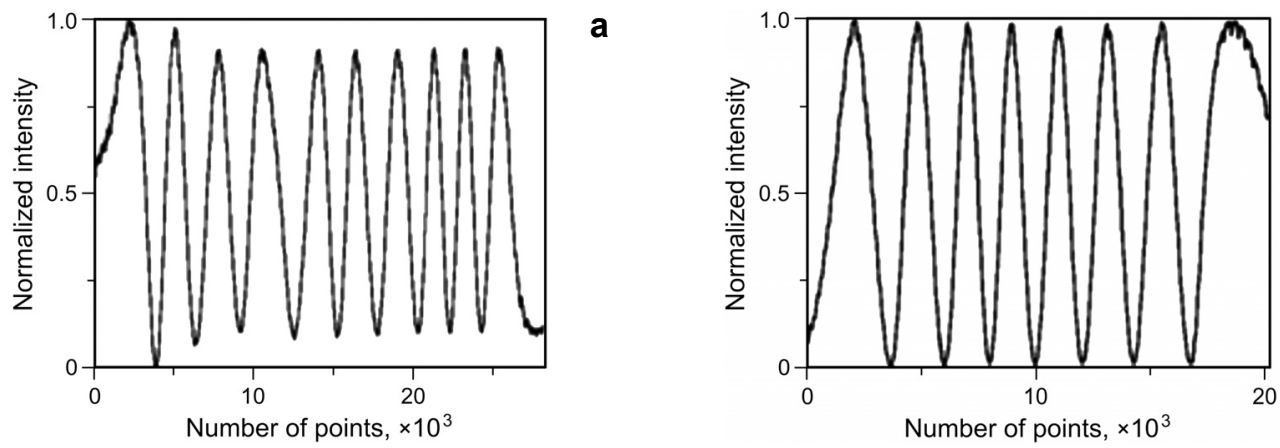

Fig. 7. Experimental dependence of intensity on longitudinal displacement of wedges for high (a) and low (b) concentration of particles.

Finding the extreme magnitudes of the distribution function parameters was based on condition of the minimum mean square deviation of theoretical magnitudes of the transversal coherence function from the observed ones (Fig. 8). For optical scheme defect correction we made some control measurements on interferometer without a sample. In ideal case, the coherence function value $\Gamma_{\perp}$ for interferometer has to be equal to 1, independently of the transversal displacement. But optical scheme defects cause interferometer noise, which we use for normalization of the obtained experimental magnitudes of the coherence function.
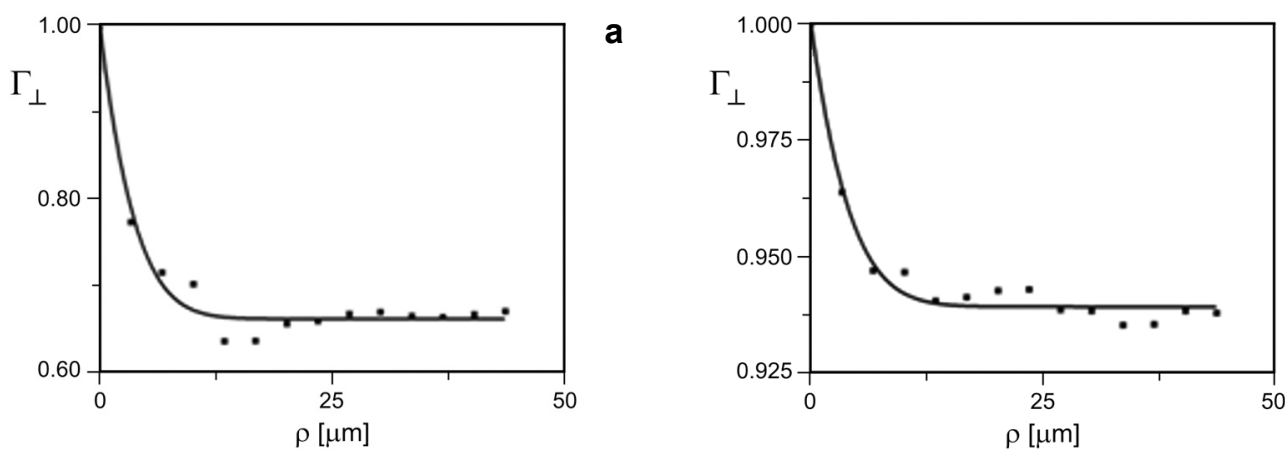

Fig. 8. Experimentally obtained magnitudes of coherence function (dots) and theoretical graph following the least-squares method (line), for samples with large (a) and small (b) concentration of particles.

Original experimental data processing provides the particle size distribution measurement with error less than $10 \%$ for each measurement in comparison with microscopical results.

Each experimental measurement provides information about distribution of particles, which are found at the observation plane. That is why, in order to receive the data on complete cement particle size distribution on a sample, we made measurements in different parts of the sample. The analysis of microscopical cement sample images shows that in different parts of the sample, the most probable particle 
T a b l e 1. Experimental data for samples with high concentration.

\begin{tabular}{cll}
\hline No. & $\sigma[\mu \mathrm{m}]$ & $\rho_{s}$ \\
\hline 1 & 5.3 & 4.0 \\
2 & 6.1 & 4.0 \\
3 & 6.1 & 3.7 \\
4 & 4.1 & 3.7 \\
5 & 6.1 & 3.7 \\
6 & 5.6 & 3.7 \\
7 & 6.0 & 3.7 \\
8 & 7.0 & 3.8 \\
9 & 6.7 & 4.0 \\
10 & 4.8 & 3.7 \\
\hline
\end{tabular}

$\mathrm{T}$ a b 1 e 2. Experimental data for samples with low concentration.

\begin{tabular}{ccl}
\hline No. & $\sigma[\mu \mathrm{m}]$ & $\rho_{s}$ \\
\hline 1 & 5.8 & 18.4 \\
2 & 6.3 & 18.3 \\
3 & 5.4 & 16.4 \\
4 & 6.7 & 17.4 \\
5 & 4.1 & 18.9 \\
6 & 5.9 & 17.7 \\
7 & 5.8 & 17.4 \\
8 & 10.6 & 16.6 \\
9 & 7.9 & 16.0 \\
10 & 4.9 & 15.7 \\
\hline
\end{tabular}

size varies from 4 to $8 \mu \mathrm{m}$. This limit was an assessment criterion of reliability for the result obtained by one measurement for our samples.

There were 10 measurements performed on samples with high and 10 with low concentration. Distributive parameters and parameter $\rho_{s}$ were found from the experimental data obtained. The results are given in Tabs. 1 and 2, respectively.

According to statistics rules, measurement results in limited quantity are described by Student's distribution. The interval with certain confidence level is defined as [11]:

$$
\bar{x} \pm \Delta(D, P, N)
$$

where sample standard deviation $D$ :

$$
D=\sqrt{\frac{1}{N-1} \sum_{n}\left(x_{n}-\bar{x}\right)^{2}}
$$


average value $\bar{x}$ :

$$
\bar{x}=\frac{1}{N} \sum_{n} x_{n}
$$

and $P$ - confidence level, $N$ - number of measurements, $x_{n}$ - result of the $n$-th measurement. For $P=0.95$ and $N=10$, the spread in the values is defined as:

$$
\Delta(D, 0.95,10)=2.262 D
$$

Processing of obtained results gives us such confidence intervals with confidence level $P=0.95$ : for samples with high concentration $\sigma=3.9-7.7 \mu \mathrm{m}$, and for samples with low concentration $\sigma=3.7-9.0 \mu \mathrm{m}$.

\section{Conclusions}

As can be seen from experimental results and calculations, confidence intervals for samples with high concentration of particles are found in reliable limits. For samples with low concentration confidence intervals slightly overstep the reliable limits. So, upon further decreasing of cement particles concentration, reliable determination of distribution for samples with low concentration is complicated. Spread in calculated values of distributive parameter $\sigma$ arises from irregularity of particle distribution in different parts of the sample. Average experimental values of the most probable cement particle size $\sigma=5.6 \mu \mathrm{m}$ for samples with high and $\sigma=6.3 \mu \mathrm{m}$ for samples with low concentration of particles correspond to values obtained for this cement by sieve method.

Experimental technique for determination of particle size distribution function used by us weakly depends on spread in refractive index values of separate particles, contary to the laser diffraction method. Calculation technique developed by us decreases calculated coherent function dependence on interferometer noise, emission source intensity fluctuations and different cement particles overlapping image effect. The technique described allows the cement particle size distribution function to be found fast and with high reliability.

\section{References}

[1] GorskiY V.F., Plugging Materials and Solutions - Handbook, Oblpoligrafvydav, Chernivtsi, 2006, p. 524 (in Ukrainian).

[2] Lee F.M., The Chemistry of Cement and Concrete, 3rd Ed., Chemical Publishing Company, 1971.

[3] Ramachandran V.S., Beaudoin J.J., Handbook of Analytical Techniques in Concrete Science and Technology, National Research Council of Canada, Ottawa, Canada, 2001.

[4] Bulatov A.I., Danyushevskiy V.S., Plugging Materials Reference Manual, Nadra, Moscow, 1987, p. 280 (in Russian). 
[5] Ferraris C.F., Hackley V.A., Aviles A.I., Measurement of particle size distribution in portland cement powder: Analysis of ASTM round robin studies, Journal of Cement, Concrete and Aggregates (CCA) 26(2), 2004, p. CCA11920.

[6] Angelsky O.V., Maksimyak P.P., Optical correlation method for studying disperse media, Applied Optics 32 (30), 1993, pp. 6137-6141.

[7] Maksimyak P.P., Angelsky O.V., An optical correlation method for measuring particle size and concentration, Proceedings of IC Mechatronics 2000, Warsaw, Poland, pp. 466-468.

[8] Ishimaru A., Wave Propagation and Scattering in Random Media, Vol. 1, 2, Academic Press, New York, 1978.

[9] Born M., Wolf E., Principles of Optics, New York, Cambridge University Press, 1999.

[10] Gorsky M.P., MaKsimyak P.P., Maksimyak A.P., Studies of light backscattering at concrete during its hydration, Ukrainian Journal of Physical Optics 10(3), 2009, pp. 134-149.

[11] Korn G.A., Korn T.M., Mathematical Handbook for Scientists and Engineers, McGraw-Hill, New York, 1987.

Received July 10, 2009

in revised form December 21, 2009 